\title{
ADIABATIC INVARIANTS \\ FOR STRONGLY NONLINEAR DYNAMICAL SYSTEMS DESCRIBED WITH COMPLEX FUNCTIONS
}

\author{
BY \\ L. CVETICANIN \\ Faculty of Technical Sciences, V. Vlahovica 3, 21000 Novi Sad, Yugoslavia
}

\begin{abstract}
In this paper the adiabatic invariants for strongly nonlinear dynamical systems with two degrees of freedom described by complex functions are obtained. The method [1] developed for dynamical systems with one degree of freedom is extended to systems with two degrees of freedom. The method is based on Noether's theory and the use of Krylov-Bogolubov-Mitropolski (KBM) and elliptic-Krylov-Bogolubov (EKB) asymptotic techniques. The adiabatic invariants for two types of strong nonlinearities are constructed: the pure cubic nonlinearity and quasi-cubic nonlinearity. The adiabatic invariants are used to obtain the approximate solution to the equations of motion.
\end{abstract}

1. Introduction. In this study we consider the problem of finding invariants of dynamical systems the motions of which are described by complex functions. Usually these are one-mass systems with two degrees of freedom. The generalized mathematical model is

$$
\ddot{z}+g^{*}(\tau, z, \dot{z}, c c)=\varepsilon f^{*}(\tau, z, \dot{z}, c c),
$$

where the left side of the equation has a Hamiltonian structure, $z=x+i y$ is a complex deflection function, $x, y$ are coordinates, $i$ is the imaginary unit, $c c$ are the corresponding complex conjugate functions, $t$ is time, $\varepsilon$ is a small parameter, $\tau=\varepsilon t$ is slow time, $f^{*}=$ $f_{1}^{*}+i f_{2}^{*}$ is an arbitrary complex function, $\left(^{*}\right)$ indicates $d / d t,\left(^{*}\right) \equiv d^{2} / d t^{2},\left(^{\prime}\right) \equiv d / d \tau$, and $\left(^{-}\right)$indicates a complex conjugate function.

The dynamical systems analyzed in this paper are strongly nonlinear. Two different types of dynamical systems are investigated:

a) the nonlinearity is quasi-cubic and the differential equation of motion is

$$
\ddot{z}+c_{3}(\tau) z(z \bar{z})=\varepsilon f^{*}(\tau, z, \dot{z}, c c) ;
$$

b) the nonlinearity is pure cubic,

$$
\ddot{z}+c_{3}(\tau) z^{3}=\varepsilon f^{*}(\tau, z, \dot{z}, c c),
$$

where $c_{3}$ is a function of slow time.

Received June 28, 1993.

1991 Mathematics Subject Classification. Primary 70K99, 70H33.

(C)1996 Brown University 
In both systems, besides the strong nonlinearity, a weak nonlinearity also exists. It is a function of slow time, complex and conjugate deflection functions, and corresponding velocities. According to the author's knowledge the invariants of the systems with two degrees of freedom are insufficiently investigated. Some investigations were done in obtaining invariants of the uncoupled dynamical systems (see [2-4]) and "weakly" coupled systems where the coupling is realized through the existence of small nonlinearities [5]. In [6] the conservation law for a rheo-linear system with linear coupling and two degrees of freedom, which possesses Hamiltonian structure, is obtained.

In this paper the previous considerations are extended to a special group of dynamical systems with strong nonlinear couplings and a complex deflection function. The method for finding adiabatic invariants of systems with two degrees of freedom suggested in this paper represents an extension of the procedure developed by Djukic [1] for obtaining adiabatic invariants for systems with one degree of freedom. The method can be described briefly as follows.

The method is based on Noether's theory [7] and the use of Krylov-BogolubovMitropolski (KBM) [8, 9] and elliptic-Krylov-Bogolubov asymptotic techniques [10-15]. Noether's theory requires the study of the invariant properties of the Lagrangian function with respect to infinitesimal transformations of the complex deflection and time. A conservation law exists if the infinitesimal transformation of the complex deflection and time leaves the Lagrangian function invariant. The necessary condition for existence of first integrals, i.e., exact invariants, is transformed into the KBM or EKB variables. Any approximate solution of this necessary condition yields the corresponding adiabatic invariant.

The time derivatives of the invariant can be of any order with respect to the power of the small parameter $\varepsilon$. In our consideration only the first-order approximation is obtained. The procedure of higher approximations is the same, but requires more calculation.

It is indicated how the invariants may be used for forming of the approximate solutions. If the adiabatic invariants are independent (the Jacobian determinant (see [7]) is nonzero), they can be solved with respect to the KBM or EKB variables and approximate solution of the problem can be formed. It is applied to a dynamical system with strong nonlinearity and linear time-dependent parameter.

2. Noether's theory for systems described with complex functions. We may construct the Lagrangian and Hamiltonian functions for the conservative part of Eq. (1) dependent on slow time, complex and conjugate deflection functions, complex and conjugate velocity, and corresponding complex and conjugate generalized momenta:

$$
\begin{aligned}
L & =L(\tau, z, \dot{z}, c c), \\
H & =H\left(\tau, z, \rho_{z}, c c\right),
\end{aligned}
$$

where $\rho_{z}=\rho_{x}+i \rho_{y}$ is the complex generalized momenta, $\rho_{x} \equiv \frac{\partial L}{\partial \dot{x}}, \rho_{y} \equiv \frac{\partial L}{\partial \dot{y}}, \rho_{x}$ and $\rho_{y}$ are generalized momenta. The functions $L$ and $H$ have real values. 
Let us consider the transformation of the time, deflections, and momenta:

$$
\begin{aligned}
& t^{*} \cong t+\mu \sigma\left(t, \tau, z, \rho_{z}, c c\right), \\
& z^{*} \cong z+\mu \phi\left(t, \tau, z, \rho_{z}, c c\right), \\
& \bar{z}^{*} \cong \bar{z}+\mu \bar{\phi}\left(t, \tau, z, \rho_{z}, c c\right), \\
& \dot{z}^{*} \cong \dot{z}+\mu\left[\dot{\phi}-\dot{z} \dot{\sigma}+\Phi\left(t, \tau, z, \rho_{z}, c c\right)\right], \\
& \dot{\bar{z}}^{*} \cong \dot{\bar{z}}+\mu\left[\overline{\dot{\phi}}-\overline{\dot{z}} \dot{\sigma}+\bar{\Phi}\left(t, \tau, z, \rho_{z}, c c\right)\right], \\
& \rho_{z}^{*} \cong \rho_{z}+\mu \nu\left(t, \tau, z, \rho_{z}, c c\right), \\
& \bar{\rho}_{z}^{*} \cong \bar{\rho}_{z}+\mu \bar{\nu}\left(t, \tau, z, \rho_{z}, c c\right),
\end{aligned}
$$

where $\mu$ is a small parameter of the transformation and $\sigma, \phi, \Phi$, and $\nu$ are unknown functions and $\bar{\phi}, \bar{\Phi}$, and $\bar{\nu}$ are the corresponding complex conjugate functions. The infinitesimal transformations are:

$$
\begin{aligned}
& \Delta t \cong \mu \sigma, \\
& \Delta z^{*} \cong \mu \phi, \\
& \Delta \bar{z}^{*} \cong \mu \bar{\phi}, \\
& \Delta \dot{z}^{*} \cong \mu(\dot{\phi}-\dot{z} \dot{\sigma}+\Phi), \\
& \Delta \overline{\dot{z}}^{*} \cong \mu(\overline{\dot{\phi}}-\overline{\dot{z}} \dot{\sigma}+\bar{\Phi}), \\
& \Delta \rho_{z}^{*} \cong \mu \nu, \\
& \Delta \bar{\rho}_{z}^{*} \cong \mu \bar{\nu} .
\end{aligned}
$$

Let us assume that the following expression,

$$
I=\left[\frac{1}{2}\left(\rho_{z} \dot{\bar{z}}+\bar{\rho}_{z} \dot{z}\right)-H\right] d t
$$

is invariant under the transformation (7) in the sense that

$$
\Delta I=\left[\frac{1}{2}\left(\rho_{z}^{*} \overline{\dot{z}}^{*}+\bar{\rho}_{z}^{*} \dot{z}^{*}\right)-H^{*}\right] d t^{*}-\left[\frac{1}{2}\left(\rho_{z} \dot{\bar{z}}+\bar{\rho}_{z} \dot{z}\right)-H\right] d t=0
$$

where $H^{*} \equiv H^{*}\left(t^{*}, z^{*}, \rho_{z}^{*}, c c^{*}\right)$.

We note that Eq. (9) could be generalized by adding a total differential (gauge function). It turns out that we do not need this additional term. Combining (4), (5), and (6), developing the term $H^{*}$ in series around the point $\left(t, z, \rho_{z}, c c\right)$, and retaining only the terms linear in the small parameter $\nu$, we obtain

$$
\begin{array}{r}
\Delta I=\mu\left[\nu\left(\frac{1}{2} \dot{\bar{z}}-\frac{\partial H}{\partial \rho_{z}}\right)+\bar{\nu}\left(\frac{1}{2} \dot{z}-\frac{\partial H}{\partial \bar{\rho}_{z}}\right)+\frac{1}{2} \rho_{z}(\dot{\bar{\phi}}+\bar{\Phi})+\frac{1}{2} \bar{\rho}_{z}(\dot{\phi}+\Phi)\right. \\
\left.-\frac{\partial H}{\partial z} \phi-\frac{\partial H}{\partial \bar{z}} \bar{\phi}-\sigma \frac{\partial H}{\partial t}-H \dot{\sigma}\right] d t
\end{array}
$$


or in equivalent form:

$$
\begin{aligned}
\Delta I=\mu & \left\{\frac{d}{d t}\left[\frac{1}{2}\left(\rho_{z} \bar{\phi}+\bar{\rho}_{z} \phi\right)-H \sigma\right]+\frac{1}{2} \rho_{z} \bar{\Phi}+\frac{1}{2} \bar{\rho}_{z} \Phi\right. \\
& +\left(\frac{1}{2} \dot{z}-\frac{\partial H}{\partial \bar{\rho}_{z}}\right)\left(\bar{\nu}-\dot{\bar{\rho}}_{z} \sigma\right)+\left(\frac{1}{2} \dot{\bar{z}}-\frac{\partial H}{\partial \rho_{z}}\right)\left(\nu-\dot{\rho}_{z} \sigma\right) \\
& +\left(\frac{1}{2} \dot{\bar{\rho}}_{z}+\frac{\partial H}{\partial z}-\frac{1}{2} \varepsilon \bar{f}^{*}\right)(\dot{z} \sigma-\phi)+\left(\frac{1}{2} \dot{\rho}_{z}+\frac{\partial H}{\partial \bar{z}}-\frac{1}{2} \varepsilon f^{*}\right)(\dot{\bar{z}} \sigma-\bar{\phi}) \\
& \left.+\frac{1}{2} \varepsilon f^{*}(\dot{\bar{z}} \sigma-\bar{\phi})+\frac{1}{2} \varepsilon \bar{f}^{*}(\dot{z} \sigma-\phi)\right\} d t .
\end{aligned}
$$

For the real Hamiltonian function (5), which is described with complex and conjugate deflections and momenta, Hamilton's equations are

$$
\begin{aligned}
\dot{z} & =2 \frac{\partial H}{\partial \bar{\rho}_{z}}, & \dot{\bar{z}} & =2 \frac{\partial H}{\partial \rho_{z}} \\
\dot{\rho}_{z} & =-2 \frac{\partial H}{\partial \bar{z}}, & \dot{\rho}_{z} & =-2 \frac{\partial H}{\partial z}
\end{aligned}
$$

If the functions $\phi, \bar{\phi}, \sigma, \Phi$, and $\bar{\Phi}$ satisfy the relation

$$
\rho_{z} \bar{\Phi}+\bar{\rho}_{z} \Phi+\varepsilon f^{*}(\dot{\bar{z}} \sigma-\bar{\phi})+\varepsilon \bar{f}^{*}(\dot{z} \sigma-\phi)=0
$$

we may deduce the following statement from (10) and (11):

If under the infinitesimal transformation (7) the expression (8) is invariant in the sense of Eq. (10), then the quantity

$$
D=\frac{1}{2}\left(\rho_{z} \bar{\phi}+\bar{\rho}_{z} \phi\right)-H \sigma
$$

is an exact invariant for the dynamical system under consideration. The necessary conditions for the functions $\phi, \bar{\phi}$, and $\sigma$ that must be satisfied if the function $I$ is invariant under the infinitesimal transformation (7) are given by

$$
\frac{1}{2}\left(\dot{\rho}_{z} \bar{\phi}+\dot{\bar{\rho}}_{z} \phi+\rho_{z} \dot{\bar{\phi}}+\bar{\rho}_{z} \dot{\phi}\right)-\dot{H} \sigma-H \dot{\sigma}=0
$$

and Eq. (13).

3. Noether's theory in KBM variables. Let us consider the dynamical system with quasi-cubic nonlinearity described in (2). Lagrange's and Hamilton's functions are

$$
\begin{aligned}
& L=\frac{1}{2}(\dot{z} \dot{\bar{z}})-\frac{1}{4} c_{3}(\tau)(z \bar{z})^{2} \\
& H=\frac{1}{2} \rho_{z} \bar{\rho}_{z}+\frac{1}{4} c_{3}(\tau)(z \bar{z})^{2}
\end{aligned}
$$

where $\rho_{z}=\dot{z}$ and $\bar{\rho}_{z}=\dot{\bar{z}}$. 
For the single frequency case let us transform the complex variables $z, \bar{z}, \rho_{z}, \bar{\rho}_{z}$ into the KBM variables $A$ and $\psi$ (see [16]):

$$
\begin{array}{rlrl}
z & =A \exp (i \psi), \quad \bar{z} & =A \exp (-i \psi), \\
\rho_{z} & =A i \omega \exp (i \psi), \quad \bar{\rho}_{z}=-A i \omega \exp (-i \psi),
\end{array}
$$

where

$$
\begin{gathered}
\psi=\int_{0}^{t} \omega(s) d s+\theta(t), \\
\omega=A \sqrt{c_{3}},
\end{gathered}
$$

and the KBM variables are functions of time $t$. Equation (2) is now replaced by a system of two first-order differential equations

$$
\begin{aligned}
& \dot{A}=-\varepsilon \frac{A c_{3}^{\prime}}{4 c_{3}}+\frac{\varepsilon}{2 A \sqrt{c_{3}}} \operatorname{Im}[f \exp (-i \psi)], \\
& \dot{\psi}=A \sqrt{c_{3}}-\frac{\varepsilon}{A^{2} \sqrt{c_{3}}} \operatorname{Re}[f \exp (-i \psi)],
\end{aligned}
$$

where $f$ is the function $f^{*}$ with the Hamiltonian variables replaced by the KBM variables (18) and (19).

The Hamiltonian (17) in the new variables is

$$
H=\frac{3}{4} A^{4} c_{3} .
$$

Substituting (18) and (19) into (14) the first integral of motion for the dynamical system under consideration and the conditions for $I$ to be invariant are transformed in KBM variables. The exact invariant (14) is

$$
D=\frac{1}{2} A^{2} i \sqrt{c_{3}}[\exp (i \psi) \bar{\phi}-\exp (-i \psi) \phi]-\frac{3}{4} A^{4} c_{3} \sigma
$$

where the functions $\phi, \Phi, \sigma$ and the corresponding complex functions satisfy the relations (13) and (15), i.e.,

$$
\begin{aligned}
& \left\{A i \sqrt{c_{3}}[\exp (i \psi) \bar{\phi}-\exp (-i \psi) \phi]-3 \sigma A^{3} c_{3}\right\} \\
& \quad \times\left\{-\varepsilon \frac{A c_{3}^{\prime}}{4 c_{3}}+\frac{\varepsilon}{2 A \sqrt{c_{3}}} \operatorname{Im}[f \exp (-i \psi)]\right\} \\
& \quad-\frac{1}{2} A^{2} \sqrt{c_{3}}[\exp (i \psi) \bar{\phi}-\exp (-i \psi) \phi]\left\{A \sqrt{c_{3}}-\frac{\varepsilon}{A^{2} \sqrt{c_{3}}} \operatorname{Re}[f \exp (-i \psi)]\right\} \\
& +\frac{\varepsilon A^{2} i c_{3}^{\prime}}{4 \sqrt{c_{3}}}[\exp (i \psi) \bar{\phi}-\exp (-i \psi) \phi] \\
& +A^{2} i \sqrt{c_{3}}[\exp (i \psi) \dot{\bar{\phi}}-\exp (-i \psi) \dot{\phi}]-\frac{3}{4} \varepsilon A^{4} c_{3}^{\prime} \sigma-\frac{3}{4} A^{4} c_{3} \dot{\sigma}=0
\end{aligned}
$$

and

$$
\begin{array}{r}
A^{2} i \sqrt{c_{3}}[\exp (i \psi) \bar{\Phi}-\exp (-i \psi) \Phi]-\varepsilon f\left[A^{2} i \sqrt{c_{3}} \sigma \exp (-i \psi)+\bar{\phi}\right] \\
+\varepsilon \bar{f}\left[A^{2} i \sqrt{c_{3}} \sigma \exp (i \psi)-\phi\right]=0
\end{array}
$$


As is well known from the theory of first integrals, the functions $\sigma, \phi, \Phi_{2}$ and the corresponding conjugate functions may be any solutions of (26) and (27). So they define some classes of exact invariants. If Eq. (26) admits a solution in $\phi$ and $\sigma$, then the equation of motion (2) admits an exact invariant (25) in KBM variables.

We must remember that in Hamiltonian mechanics the generalized momenta are known functions of time, generalized coordinates, and generalized velocities. Hence, the transformed generalized momenta $\rho_{x}^{*}$ and $\rho_{y}^{*}$ are determined by the transformed quantities $t^{*}, z^{*}, \dot{z}^{*}$, i.e., the quantities $\nu$ and $\bar{\nu}$ in (6) and (7) are functions of $\sigma, \phi$, $\Phi$, and $c c$.

4. The adiabatic invariants for the quasi-cubic system. The possibility of finding the exact solution of Eq. (26) is strongly limited by the particular forms of the functions $c_{3}$ and $f$. For this reason we search for approximate solutions of this equation. The invariance condition $\Delta I=0$ is not fulfilled by those solutions. Here the approximate solution is proportional to $\varepsilon$ leading the $\Delta I$ proportional to $\varepsilon^{2}$.

Let us assume the solution of Eq. (26) as the following series in the small parameter $\varepsilon$ :

$$
\begin{aligned}
\sigma & =\sigma_{0}(\tau, A, \psi)+\varepsilon \sigma_{1}(\tau, A, \psi)+\varepsilon^{2}+\cdots, \\
\phi & =\phi_{0}(\tau, A, \psi)+\varepsilon \phi_{1}(\tau, A, \psi)+\varepsilon^{2}+\cdots, \\
\bar{\phi} & =\bar{\phi}_{0}(\tau, A, \psi)+\varepsilon \bar{\phi}_{1}(\tau, A, \psi)+\varepsilon^{2}+\cdots,
\end{aligned}
$$

where $\sigma_{0}, \sigma_{1}, \ldots, \phi_{0}, \phi_{1}, \ldots$ and their complex conjugates $\bar{\phi}_{0}, \bar{\phi}_{1}, \ldots$ are unknown functions. Substituting (28), (29), and (30) into (26), separating terms of the same order with respect to the small parameter $\varepsilon$, and neglecting quantities of order $\varepsilon^{2}$, we obtain the following two partial differential equations:

$$
\begin{aligned}
& \varepsilon^{0}: \quad \operatorname{Ai}\left[\exp (i \psi) \dot{\phi_{0}}-\exp (-i \psi) \dot{\phi}_{0}\right] \\
& -\frac{1}{2} A^{2} \sqrt{c_{3}}\left[\exp (i \psi) \bar{\phi}_{0}-\exp (-i \psi) \phi_{0}\right]-\frac{3}{4} A^{4} c_{3} \frac{\partial \sigma_{0}}{\partial \psi}=0 . \\
& \varepsilon^{1}: \quad\left\{A i \sqrt{c_{3}}\left[\exp (i \psi) \bar{\phi}_{0}-\exp (-i \psi) \phi_{0}\right]-3 \sigma_{0} A^{3} c_{3}\right\} \\
& \times\left\{-\frac{A c_{3}^{\prime}}{4 c_{3}}+\frac{1}{2 A \sqrt{c_{3}}} \operatorname{Im}[f \exp (-i \psi)]\right\} \\
& -\frac{1}{2} A^{3} c_{3}\left[\exp (i \psi) \bar{\phi}_{1}-\exp (-i \psi) \phi_{1}\right] \\
& +\frac{1}{2} A^{2} \sqrt{c_{3}}\left[\exp (i \psi) \bar{\phi}_{0}-\exp (-i \psi) \phi_{0}\right] \frac{1}{A^{2} \sqrt{c_{3}}} \operatorname{Re}[f \exp (-i \psi)] \\
& +\frac{A^{2} i c_{3}^{\prime}}{4 \sqrt{c_{3}}}\left[\exp (i \psi) \bar{\phi}_{0}-\exp (-i \psi) \phi_{0}\right] \\
& +A^{2} i \sqrt{c_{3}}\left[\exp (i \psi) \dot{\bar{\phi}}_{1}-\exp (-i \psi) \dot{\phi}_{1}\right]-\frac{3}{4} A^{4} c_{3}^{\prime} \sigma_{0}-\frac{3}{4} A^{5} c_{3} \sqrt{c_{3}} \frac{\partial \sigma_{1}}{\partial \psi} \\
& -\frac{3}{4} A^{4} c_{3} \frac{\partial \sigma_{0}}{\partial A}\left\{-\frac{A c_{3}^{\prime}}{4 c_{3}}+\frac{1}{2 A \sqrt{c_{3}}} \operatorname{Im}[f \exp (-i \psi)]\right\}-\frac{3}{4} A^{4} c_{3} \frac{\partial \sigma_{0}}{\partial \tau} \\
& +\frac{3}{4} A^{2} \sqrt{c_{3}} \frac{\partial \sigma_{0}}{\partial \psi} \operatorname{Re}[f \exp (-i \psi)]=0 .
\end{aligned}
$$


In these nonlinear equations the functions $\sigma_{0}, \sigma_{1}, \phi_{0}, \phi_{1}$, and $c c$ are unknowns. We stress that these functions may be any particular solutions of Eqs. (31) and (32). There are four independent unknown functions and only two equations. This enables us to choose two arbitrary functions and then to find the other two functions from the equations.

One of the simplest forms of the functions that satisfy Eqs. (31) and (32) is

$$
\begin{gathered}
\phi_{0}=0, \quad \phi_{1}=0 \quad\left(\bar{\phi}_{0}=0, \quad \bar{\phi}_{1}=0\right), \\
\sigma_{1}=\frac{1}{A \sqrt{c_{3}}}\left\{\frac{1}{4} \frac{\sigma_{0}(A, \tau)}{c_{3}^{\prime}}\left(A \frac{\partial \sigma_{0}}{\partial A}-4 c_{3} \frac{\partial \sigma_{0}}{\partial c_{3}}\right) \psi\right. \\
-\frac{2}{A^{2} \sqrt{c_{3}}}\left(\sigma_{0}+\frac{1}{4} A \frac{\partial \sigma_{0}}{\partial A}\right) \int \operatorname{Im}[f \exp (-i \psi) d \psi\}
\end{gathered}
$$

where $\sigma_{0}$ is an arbitrary function of the corresponding variables. According to (25) and (33)-(35) the adiabatic invariant is

$$
D=-\frac{3}{4} A^{4} c_{3}\left(\sigma_{0}+\varepsilon \sigma_{1}\right) .
$$

We emphasize that in the adiabatic invariant (36) the function $\sigma_{0}$ remains completely arbitrary. Now we select the function $\sigma_{0}$ in such a way as to give some special forms of the adiabatic invariant.

a) For

$$
\sigma_{0}=\frac{1}{A \sqrt{c_{3}}},
$$

the adiabatic invariant has the form of Born's invariant, where the leading term is energy divided by the "frequency". It is

$$
D_{1}=\frac{H}{\omega}+\varepsilon\left\{\frac{3 A^{2} c_{3}^{\prime}}{16 c_{3}} \psi-\frac{9}{8 \sqrt{c_{3}}} \int \operatorname{Im}[f \exp (-i \psi) d \psi\} .\right.
$$

b) For the case in which $\sigma_{0}$ is taken to be

$$
\sigma_{0}=1
$$

the adiabatic invariant is of energy type

$$
D_{2}=H+\varepsilon\left\{\frac{3}{2} A \int \operatorname{Im}[f \exp (-i \psi) d \psi\} .\right.
$$

c) A special form of adiabatic invariant is obtained for

$$
\sigma_{0}=-\frac{1}{A^{4}}
$$

and it is

$$
D_{3}=\frac{3}{4} c_{3}-\frac{3}{4} \varepsilon A \frac{c_{3}^{\prime}}{\sqrt{c_{3}}} \psi
$$




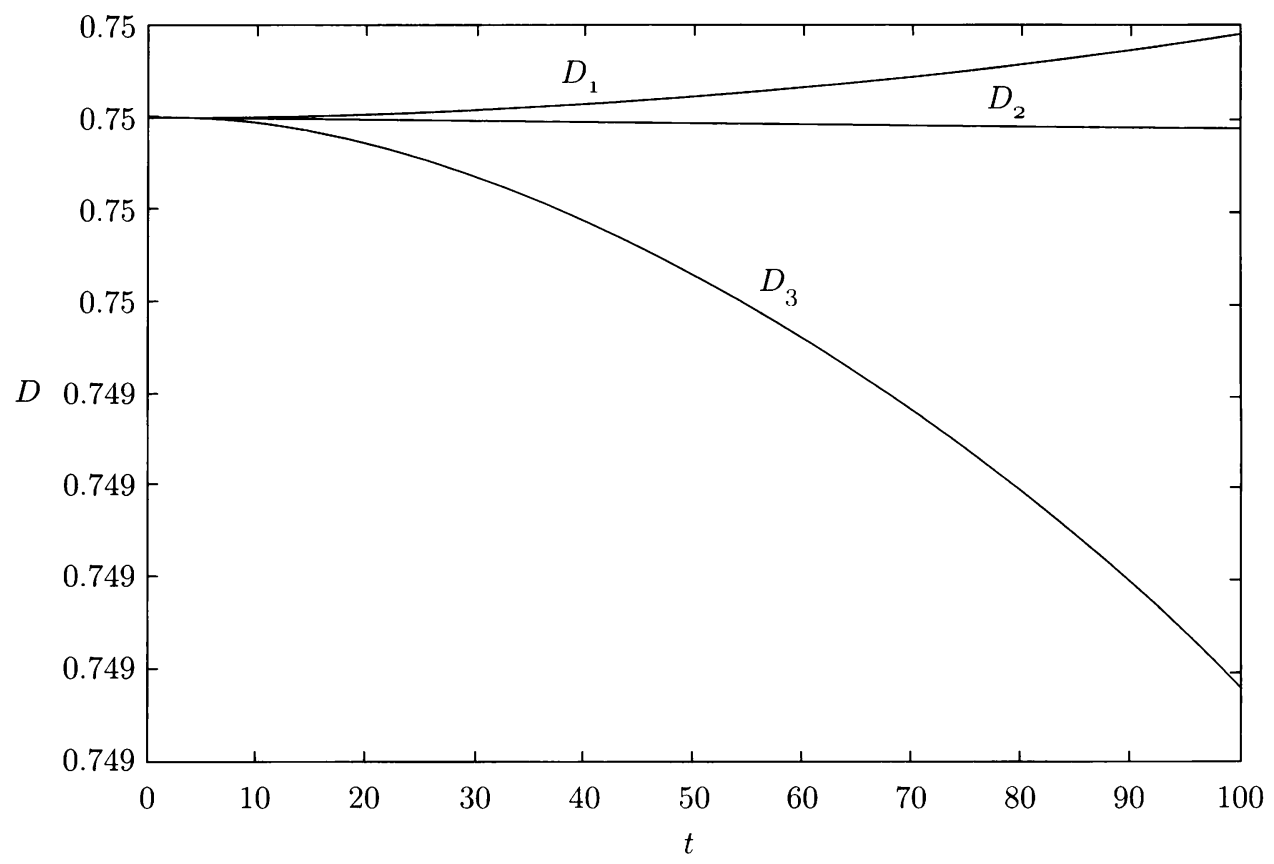

FIG. 1. Adiabatic invariants for the quasi-cubic oscillator

EXAMPLE. Let us consider the case when the perturbing function is

$$
f^{*}=0
$$

The adiabatic invariants are

$$
\begin{aligned}
& D_{1}=\frac{3}{4} A^{3} \sqrt{c_{3}}+\varepsilon \frac{3 A^{2} c_{3}^{\prime}}{16 c_{3}} \psi, \\
& D_{2}=\frac{3}{4} A^{4} c_{3}
\end{aligned}
$$

and the third invariant $D_{3}$ is given by (42).

In Fig. 1 the adiabatic invariants are plotted. The initial amplitude and phase angle are $A_{0}=1$ and $\psi_{0}=0$, respectively. $c_{3}$ is a linear function of slow time, i.e.,

$$
c_{3}=1+\tau
$$

It can be seen that all of the adiabatic invariants are almost constant.

The adiabatic invariants are independent and the Jacobian determinant of Eqs. (42), (44), and (45) is nonzero. The adiabatic invariants (44) and (45) describe the motion of the system. Using the algebraic equations (44) and (45) the KBM variables are

$$
A=A_{0}\left(\frac{c_{30}}{c_{3}}\right)^{1 / 4}
$$




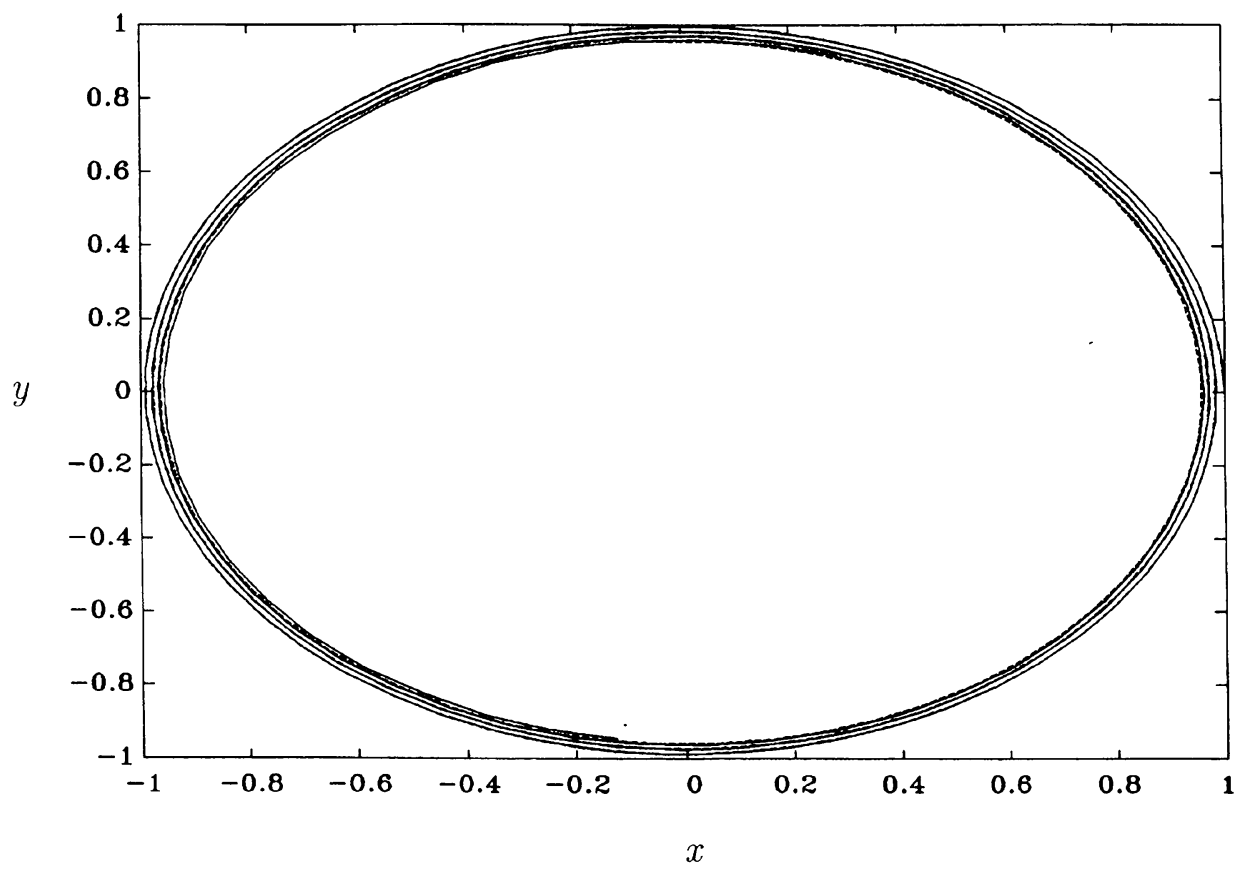

FIG. 2. Solutions of the motion for the quasi-cubic oscillator in the $(x, y)$-plane. --exact numerical solution; - - -asymptotic solution

and

$$
\psi=\frac{4 A_{0}}{\varepsilon c_{3}^{\prime}}\left[\sqrt{c_{3}^{3}}-\sqrt[4]{c_{3}^{5} c_{30}}\right] .
$$

For the previous values of the parameters the solution in the $(x, y)$-plane is plotted (Fig. 2 ). The approximate solution is compared with the exact numerical solution. Comparing the numerical and approximate solutions it is concluded that the difference is negligible for small values of the parameter $\varepsilon$.

5. Noether's theory in EKB variables. We consider the dynamical system with pure cubic nonlinearity described by (3). Lagrange's and Hamilton's functions are

$$
\begin{aligned}
L & =\frac{1}{2}\left(\dot{z}^{2}+\dot{\bar{z}}^{2}\right)-\frac{1}{8} c_{3}(\tau)\left(z^{4}+\bar{z}^{4}\right), \\
H & =\frac{1}{2}\left(\rho_{z}^{2}+\bar{\rho}_{z}^{2}\right)+\frac{1}{8} c_{3}(\tau)\left(z^{4}+\bar{z}^{4}\right),
\end{aligned}
$$

where $\rho_{z}=\dot{\bar{z}}$ and $\bar{\rho}_{z}=\dot{z}$.

We transform the complex variables $z, \bar{z}, \rho_{z}, \bar{\rho}_{z}$ into the EKB variables $A$ and $\psi$ (see $[10])$ :

$$
\begin{aligned}
z & =A[\mathrm{cn}(\psi, 1 / 2)+i \operatorname{sn}(\psi, 1 / 2)] \equiv A(\mathrm{cn}+i \mathrm{sn}), \\
\bar{z} & =A(\mathrm{cn}-i \mathrm{sn}) \\
\dot{z} & =\bar{\rho}_{z}=A \omega i \operatorname{dn}(\mathrm{cn}+i \mathrm{sn}), \\
\dot{\bar{z}} & =\rho_{z}=-A \omega i \operatorname{dn}(\mathrm{cn}-i \mathrm{sn}),
\end{aligned}
$$


where sn, cn, and dn are the Jacobian elliptic functions [17],

$$
\begin{gathered}
\psi=\int_{0}^{t} \omega(s) d s+\theta(t), \\
\omega=A \sqrt{c_{3}(\tau)}
\end{gathered}
$$

and the EKB variables are functions of time $t$. Equation (3) is now replaced by a system of two first-order differential equations,

$$
\begin{gathered}
\dot{A}=-\varepsilon \frac{A c_{3}^{\prime}}{4 c_{3}} d n^{2}-\frac{\varepsilon}{A \sqrt{c_{3}}} \frac{\mathrm{sn} \mathrm{cn}}{\mathrm{dn}^{2}} \operatorname{Re}[f(\mathrm{cn}-i \mathrm{sn})]+\frac{\varepsilon}{2 A \sqrt{c_{3}}} \operatorname{Im}[f(\mathrm{cn}-i \mathrm{sn})], \\
\dot{\psi}=A \sqrt{c_{3}}-\frac{\varepsilon}{A^{2} \sqrt{c_{3}} \mathrm{dn}^{2}} \operatorname{Re}[f(\mathrm{cn}-i \mathrm{sn})]
\end{gathered}
$$

where $f$ is the function $f^{*}$ in which the Hamiltonian variables are replaced by the EKB variables $A$ and $\psi$.

The Hamiltonian (50) in the new variables (51) and (52) is

$$
H=-\frac{1}{4} A^{4} c_{3}
$$

Substituting (51) and (52) into (14) the first integral of the motion for the dynamical system under consideration in EKB variables is obtained. It is

$$
D=\frac{1}{4} A^{4} c_{3} \sigma-\frac{1}{2} A^{2} i \sqrt{c_{3}} \operatorname{dn}[(\mathrm{cn}-i \mathrm{sn}) \bar{\phi}-(\mathrm{cn}+i \mathrm{sn}) \phi]
$$

where the functions $\phi, \Phi, \sigma$ and the corresponding complex functions satisfy the relations (13) and (15), i.e.,

$$
\begin{aligned}
& A^{2} i \sqrt{c_{3}} \operatorname{dn}[(\mathrm{cn}+i \mathrm{sn}) \Phi-(\mathrm{cn}-i \mathrm{sn}) \bar{\Phi}]-\varepsilon f\left[A^{2} i \sqrt{c_{3}} \mathrm{dn}(\mathrm{cn}-i \mathrm{sn}) \sigma-\bar{\phi}\right] \\
& +\varepsilon \bar{f}\left[A^{2} i \sqrt{c_{3}} \operatorname{dn}(\mathrm{cn}+i \mathrm{sn}) \sigma-\phi\right]=0, \\
& \frac{1}{4} A^{4} c_{3} \dot{\sigma}+\frac{1}{4} A^{4} \dot{c}_{3} \sigma-\frac{1}{4} A^{2} \frac{\dot{c}_{3}}{\sqrt{c_{3}}} \operatorname{dn}[(\mathrm{cn}-i \mathrm{sn}) \bar{\phi}-(\mathrm{cn}+i \mathrm{sn}) \phi] \\
& -\frac{1}{2} A^{2} i \sqrt{c_{3}} \mathrm{dn}[(\mathrm{cn}-i \mathrm{sn}) \dot{\bar{\phi}}-(\mathrm{cn}+i \mathrm{sn}) \dot{\phi}] \\
& -\left\{A i \sqrt{c_{3}} \operatorname{dn}[(\mathrm{cn}-i \mathrm{sn}) \bar{\phi}-(\mathrm{cn}+i \mathrm{sn}) \phi]-\frac{1}{4} A^{3} c_{3} \sigma\right\} \\
& \times\left\{-\varepsilon \frac{A c_{3}^{\prime}}{4 c_{3}} \mathrm{dn}^{2}-\frac{\varepsilon}{A \sqrt{c_{3}}} \frac{\mathrm{sn} \mathrm{cn}}{\mathrm{dn}^{2}} \operatorname{Re}[f(\mathrm{cn}-i \mathrm{sn})]+\frac{\varepsilon}{2 A \sqrt{c_{3}}} \operatorname{Im}[f(\mathrm{cn}-i \mathrm{sn})]\right\} \\
& +\left\{A^{2} i \sqrt{c_{3}} \mathrm{sn} \operatorname{cn}[(\mathrm{cn}-i \mathrm{sn}) \bar{\phi}-(\mathrm{cn}+i \mathrm{sn}) \phi]\right. \\
& \left.+\frac{1}{2} A^{2} \sqrt{c_{3}} \mathrm{dn}^{2}[(\mathrm{cn}-i \mathrm{sn}) \bar{\phi}-(\mathrm{cn}+i \mathrm{sn}) \phi]\right\} \\
& \times\left\{A \sqrt{c_{3}}-\frac{\varepsilon}{A^{2} \sqrt{c_{3}} \mathrm{dn}^{2}} \operatorname{Re}[f(\mathrm{cn}-i \mathrm{sn})]\right\}=0 \text {. }
\end{aligned}
$$


6. Adiabatic invariants for pure cubic systems. We repeat the method described in the previous section. We assume the series (28)-(30), substitute them into the relations (59) and (60), and separate terms of the same order of $\varepsilon$. It follows that

$$
\begin{aligned}
& \varepsilon^{0}: \quad \frac{1}{2} i \operatorname{dn}\left[(\mathrm{cn}-i \mathrm{sn}) \frac{\partial \bar{\phi}_{0}}{\partial \psi}-(\mathrm{cn}+i \mathrm{sn}) \frac{\partial \phi_{0}}{\partial \psi}\right] \\
& +\left(i \mathrm{sn} \mathrm{cn}+\frac{1}{2} \mathrm{dn}^{2}\right)\left[(\mathrm{cn}-i \mathrm{sn}) \bar{\phi}_{0}-(\mathrm{cn}+i \mathrm{sn}) \phi_{0}\right] \\
& -\frac{1}{4} A^{2} \sqrt{c_{3}} \frac{\partial \sigma_{0}}{\partial \psi}=0 \\
& \varepsilon: \quad \frac{1}{2} i \operatorname{dn} A^{2} \sqrt{c_{3}}\left[(\mathrm{cn}-i \mathrm{sn}) \frac{\partial \bar{\phi}_{0}}{\partial \psi}-(\mathrm{cn}+i \mathrm{sn}) \frac{\partial \phi_{0}}{\partial \psi}\right]\left\{\frac{1}{A^{2} \sqrt{c_{3}} \mathrm{dn}^{2}} \operatorname{Re}[f(\mathrm{cn}-i \mathrm{sn})]\right\} \\
& +\frac{1}{4} A^{5} c_{3} \sqrt{c_{3}} \frac{\partial \sigma_{1}}{\partial \psi}-\frac{1}{2} i \mathrm{dn} A^{3} c_{3}\left[(\mathrm{cn}-i \mathrm{sn}) \frac{\partial \bar{\phi}_{1}}{\partial \psi}-(\mathrm{cn}+i \mathrm{sn}) \frac{\partial \phi_{1}}{\partial \psi}\right] \\
& +\frac{1}{2} i \operatorname{dn} A^{2} \sqrt{c_{3}}\left[(\mathrm{cn}-i \mathrm{sn}) \frac{\partial \bar{\phi}_{0}}{\partial A}-(\mathrm{cn}+i \mathrm{sn}) \frac{\partial \phi_{0}}{\partial A}\right] \\
& \times\left\{\frac{A c_{3}^{\prime}}{4 c_{3}} \mathrm{dn}^{2}+\frac{1}{A \sqrt{c_{3}}} \frac{\mathrm{sn} c \mathrm{n}}{\mathrm{dn}^{2}} \operatorname{Re}[f(c n-i \mathrm{sn})]-\frac{1}{2 A \sqrt{c_{3}}} \operatorname{Im}[f(\mathrm{cn}-i \mathrm{sn})]\right\} \\
& -\frac{1}{2} i \operatorname{dn} A^{2} \sqrt{c_{3}} c_{3}^{\prime}\left[(\mathrm{cn}-i \mathrm{sn}) \frac{\partial \bar{\phi}_{0}}{\partial c_{3}}-(\mathrm{cn}+i \mathrm{sn}) \frac{\partial \phi_{0}}{\partial c_{3}}\right] \\
& -\frac{1}{4 \sqrt{c_{3}}} A^{2} c_{3}^{\prime} i \operatorname{dn}\left[(\mathrm{cn}-i \mathrm{sn}) \bar{\phi}_{0}-(\mathrm{cn}+i \mathrm{sn}) \phi_{0}\right]+\frac{1}{4} A^{4} c_{3}^{\prime} \sigma_{0} \\
& +\frac{1}{4} A^{4} c_{3} c_{3}^{\prime} \frac{\partial \sigma_{0}}{\partial c_{3}}+\frac{1}{4} A^{4} c_{3} \frac{\partial \sigma_{0}}{\partial A}\left\{-\frac{A c_{3}^{\prime}}{4 c_{3}} \mathrm{dn}^{2}\right. \\
& \left.-\frac{1}{A \sqrt{c_{3}}} \frac{\mathrm{sn} \mathrm{cn}}{\mathrm{dn}^{2}} \operatorname{Re}[f(\mathrm{cn}-i \mathrm{sn})]+\frac{1}{2 A \sqrt{c_{3}}} \operatorname{Im}[f(\mathrm{cn}-i \mathrm{sn})]\right\} \\
& -\frac{1}{4} A^{4} c_{3} \frac{\partial \sigma_{0}}{\partial \psi} \frac{1}{A^{2} \sqrt{c_{3}} \mathrm{dn}^{2}} \operatorname{Re}[f(\mathrm{cn}-i \mathrm{sn})] \\
& +\left\{A i \sqrt{c_{3}} \mathrm{dn}\left[(\mathrm{cn}-i \mathrm{sn}) \bar{\phi}_{0}-(\mathrm{cn}+i \mathrm{sn}) \phi_{0}\right]-\frac{1}{4} A^{3} c_{3} \sigma_{0}\right\} \\
& \times\left\{\frac{A c_{3}^{\prime}}{4 c_{3}} \mathrm{dn}^{2}+\frac{1}{A \sqrt{c_{3}}} \frac{\mathrm{sn} \mathrm{cn}}{\mathrm{dn}^{2}} \operatorname{Re}[f(\mathrm{cn}-i \mathrm{sn})]-\frac{1}{2 A \sqrt{c_{3}}} \operatorname{Im}[f(\mathrm{cn}-i \mathrm{sn})]\right\} \\
& -A^{3} c_{3}\left[i \mathrm{sn} \mathrm{cn}+\frac{1}{2} \mathrm{dn}^{2}\right]\left[(\mathrm{cn}-i \mathrm{sn}) \bar{\phi}_{1}-(\mathrm{cn}+i \mathrm{sn}) \phi_{1}\right] \\
& +\frac{1}{\mathrm{dn}^{2}} \operatorname{Re}[f(\mathrm{cn}-i \mathrm{sn})]\left[i \mathrm{sn} \mathrm{cn}+\frac{1}{2} \mathrm{dn}^{2}\right]\left[(\mathrm{cn}-i \mathrm{sn}) \bar{\phi}_{0}-(\mathrm{cn}+i \mathrm{sn}) \phi_{0}\right]=0 \text {. }
\end{aligned}
$$

Solving these equations with respect to $\sigma_{0}, \sigma_{1}, \phi_{0}, \phi_{1}$, and $c c$ we have

$$
\phi_{0}=\phi_{1}=0 \quad\left(\bar{\phi}_{0}=\bar{\phi}_{1}=0\right)
$$




$$
\begin{gathered}
\sigma_{0}=\sigma_{0}(\tau, A) \\
\sigma_{1}=\frac{1}{A \sqrt{c_{3}}}\left\{( \sigma _ { 0 } + A \frac { \partial \sigma _ { 0 } } { \partial A } ) \int \left[\frac{A c_{3}^{\prime}}{4 c_{3}} \mathrm{dn}^{2}+\frac{1}{A \sqrt{c_{3}}} \frac{\mathrm{sn} \operatorname{cn}}{\mathrm{dn}^{2}} \operatorname{Re}[f(\mathrm{cn}-i \mathrm{sn})]\right.\right. \\
\left.\left.-\frac{1}{2 A \sqrt{c_{3}}} \operatorname{Im}[f(\mathrm{cn}-i \mathrm{sn})]\right] d \psi-A c_{3}^{\prime}\left(\frac{\partial \sigma_{0}}{\partial c_{3}}-\frac{1}{c_{3}} \sigma_{0}\right) \psi\right\}
\end{gathered}
$$

where $\sigma_{0}$ is an arbitrary function of its arguments. Now combining (63)-(65) we find the quantity $D$ to be

$$
D=\frac{1}{4} A^{4} c_{3}\left(\sigma_{0}+\varepsilon \sigma_{1}\right)
$$

The quantity $D$ is the adiabatic invariant for the dynamical system for which the equation of motion is given by (3) or (55) and (56). Let us assume the arbitrary function $\sigma_{0}$ to be

$$
\sigma_{0}=1
$$

Then the adiabatic invariant is

$$
\begin{aligned}
D_{1}=H+\varepsilon H \frac{1}{A \sqrt{c_{3}}} & \left\{\int \left[\frac{A c_{3}^{\prime}}{4 c_{3}} \mathrm{dn}^{2}+\frac{1}{A \sqrt{c_{3}}} \frac{\mathrm{sn} \operatorname{cn}}{\mathrm{dn}^{2}} \operatorname{Re}[f(\mathrm{cn}-i \mathrm{sn})]\right.\right. \\
& \left.\left.-\frac{1}{2 A \sqrt{c_{3}}} \operatorname{Im}[f(\mathrm{cn}-i \mathrm{sn})]\right] d \psi+A c_{3}^{\prime} \frac{1}{c_{3}} \psi\right\} .
\end{aligned}
$$

It represents an energy-type adiabatic invariant. For

$$
\sigma_{0}=\frac{1}{A \sqrt{c_{3}}}
$$

the classical type of invariant

$$
D_{2}=\frac{H}{\omega}+\varepsilon \frac{H}{\varepsilon} \frac{c_{3}^{\prime}}{\sqrt{c_{3}}}\left(\frac{c_{3}^{\prime}}{2}+\frac{1}{c_{3}}\right) \psi
$$

is obtained. The adiabatic invariant

$$
D_{3}=\frac{c_{3}^{2} A^{3}}{4}
$$

is obtained by assuming

$$
\sigma_{0}=\frac{c_{3}}{A} .
$$

EXAMPLE. If $f^{*}=0$, the adiabatic invariants are

$$
D_{1}=H+\varepsilon H \frac{1}{\sqrt{c_{3}}} c_{3}^{\prime} \frac{1}{c_{3}} \psi
$$




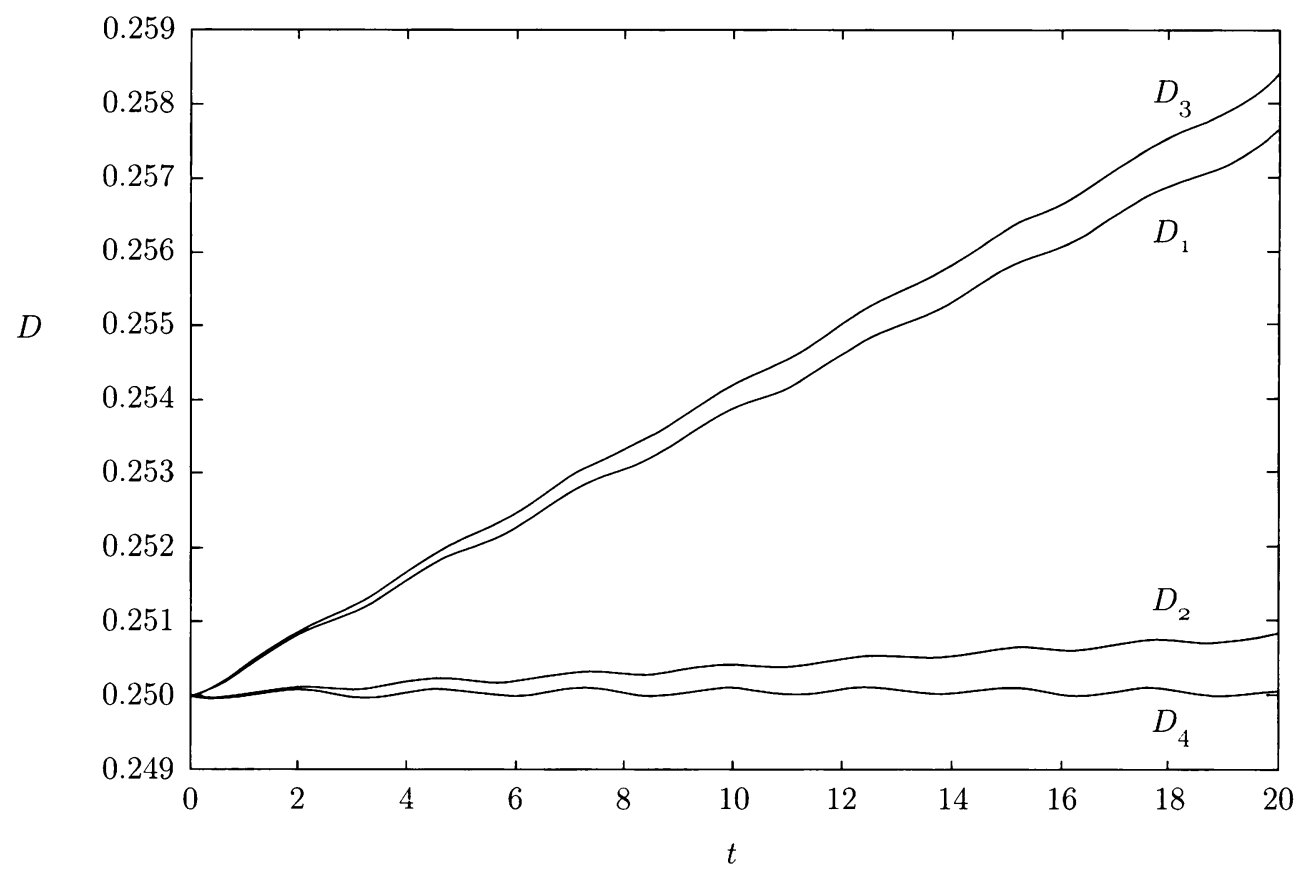

FIG. 3. Adiabatic invariants for the cubic oscillator

and the invariants $D_{2}$ and $D_{3}$ are unchanged. Any linear combination of the adiabatic invariants gives a new adiabatic invariant, for example,

$$
D_{4}=D_{1}+D_{2}-D_{3}
$$

In Fig. 3 the adiabatic invariants are plotted. The initial conditions are $A=A_{0}=1$ and $\psi=\psi_{0}=0$. The time-variable function is the same as in the previous example (see Eq. (46)). It can be seen that the invariants $D_{2}$ and $D_{4}$ are almost constant and $D_{3}$ and $D_{4}$ are slowly increasing. $D_{2}$ and $D_{4}$ are independent and these algebraic relations enable us to write the EKB variables as

$$
\begin{gathered}
A=A_{0} \sqrt[6]{c_{30} / c_{3}} \\
\psi=\frac{\sqrt{c_{3}}}{\varepsilon c_{3}^{\prime}}\left[\left(\frac{A_{0}}{A}\right)^{4}-\left(c_{3}+\frac{\sqrt{c_{3}}}{A}-\frac{c_{3}^{2}}{A}\right)\right] .
\end{gathered}
$$

In Fig. 4 (see p. 420) the approximate analytical solutions and the exact numerical solution are plotted in the $(x, y)$-plane. The difference between the solutions is negligible. 


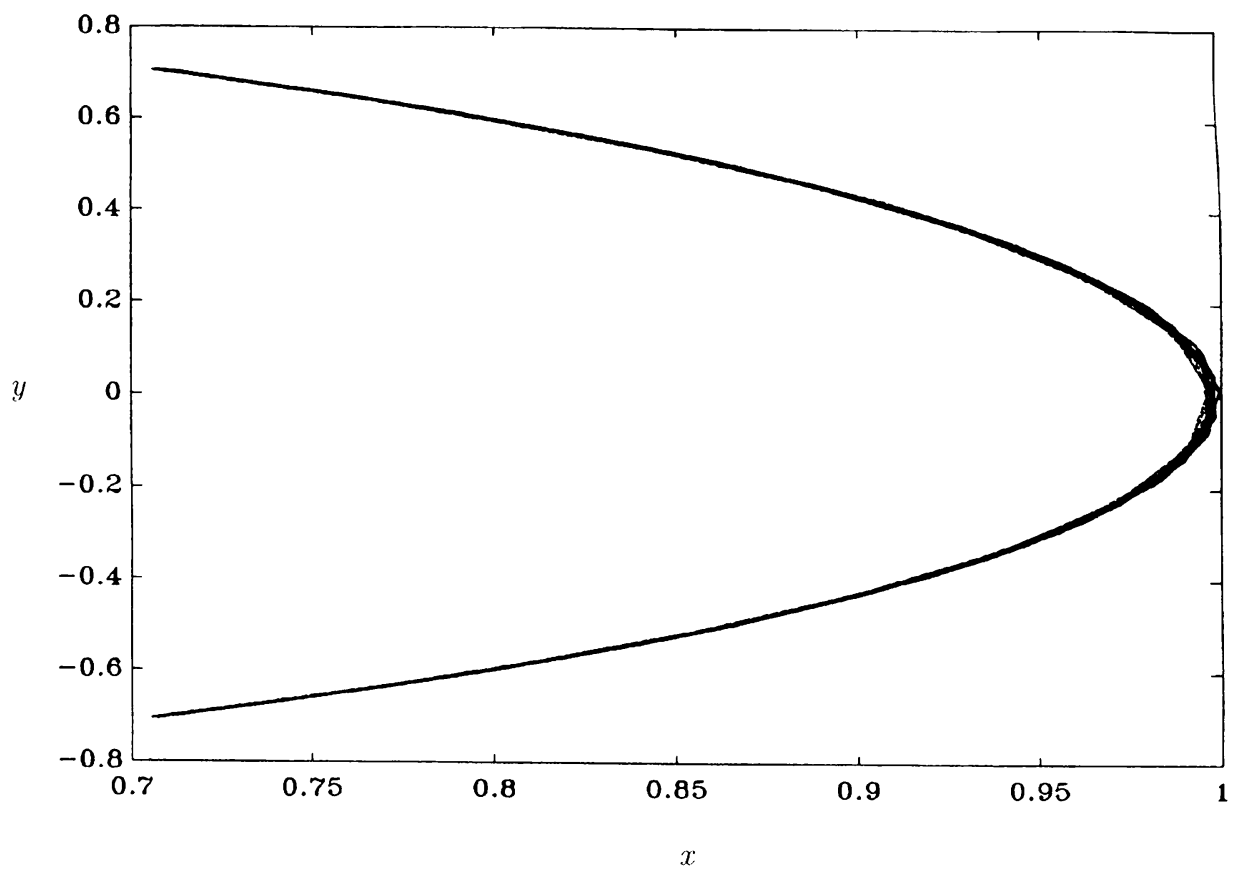

FIG. 4. Solutions of the motion for the cubic oscillator in the $(x, y)$ plane. -Exact numerical solution; - - -asymptotic solution

Acknowledgments. I thank Prof. Dj. Djukic for his valuable suggestions and comments. These investigations were supported by SANU and the Government of Serbia.

\section{REFERENCES}

[1] Dj. S. Djukic, Adiabatic invariants for dynamical systems with one degree of freedom, Internat. J. Non-Linear Mech. 16, 489 498 (1981)

[2] P. G. L. Leach, Invariants and wave functions for some time-dependent harmonic oscillator-type Hamiltonians, J. Math. Phys. 18, 1902-1907 (1977)

[3] N. J. Gunther and P. G. L. Leach, Generalized invariants for the time-dependent harmonic oscillator, J. Math. Phys. 18, 572-576 (1977)

[4] W. Sarlet and L. Y. Bahar, A direct construction of first integrals for certain non-linear dynamical systems, Internat. J. Non-Linear Mech. 15, 133-146 (1980)

[5] L. Cveticanin, Adiabatic invariants of dynamical systems with two degrees of freedom, Internat. J. Non-linear Mech. 29, 799-808 (1994)

[6] B. D. Vujanovic, Conservation laws of rheo-linear dynamical systems with one- and two-degreesof-freedom, Internat. J. Non-Linear Mech. 27, 309-322 (1992)

[7] B. D. Vujanovic and S. E. Jones, Variational Methods in Nonconservative Phenomena, Academic Press, New York, 1989, p. 370

[8] L. Cveticanin, Approximate analytical solutions to a class of non-linear equations with complex functions, J. Sound Vibration 157, no. 2, 289-302 (1992)

[9] N. N. Bogolubov and Ju. A. Mitropolski, Asimptoticheskie metodi v teorii nelinejnih kolebanij, Gos. Fiz. Mat. Lit., Moscow, 1963

[10] L. Cveticanin, An approximate solution of a coupled differential equation with variable parameter, Trans. ASME Ser. E. J. Appl. Mech. 60, no. 1, 214-217 (1993)

[11] S. Bravo Yuste and J. Diaz Bejarano, Construction of approximate analytical solutions to a new class of nonlinear oscillator equations, J. Sound Vibration 110, 347-350 (1986) 
[12] S. Bravo Yuste and J. Diaz Bejarano, Improvement of a Krylov-Bogoliubov method that uses Jacobi elliptic functions, J. Sound Vibration 139, 151-163 (1990)

[13] V. T. Coppola and R. H. Rand, Averaging using elliptic functions: Approximation of limit cycles, Acta Mechanica 81, 125-142 (1990)

[14] S. Bravo Yuste, Quasi-pure-cubic oscillators studied using a Krylov-Bogoliubov method, J. Sound Vibration 158, 267-275 (1992)

[15] S. Bravo Yuste, On Duffing oscillators with slowly varying parameters, Internat J. Non-Linear Mech. 26, 671-677 (1991)

[16] L. Cveticanin, An approximate solution for a system of two coupled differential equations, J. Sound Vibration 152, 375-380 (1992)

[17] M. Abramowitz and I. A. Stegun, Spravochnik po specialynyim funkcijam, Moscow, Nauka, 1979 